\title{
(C) OPEN ACCESS \\ Lupus-associated atypical memory B cells are mTORC1-hyperactivated and functionally dysregulated
}

\author{
Chunmei Wu, ${ }^{1,2}$ Qiong Fu, ${ }^{1}$ Qiang Guo, ${ }^{1}$ Sheng Chen, ${ }^{1}$ Shyamal Goswami, ${ }^{2}$ \\ Shuhui Sun, ${ }^{1}$ Teng Li, ${ }^{2}$ Xingjian Cao, ${ }^{3}$ Fuying Chu, ${ }^{3}$ Zechuan Chen, ${ }^{2}$ Mei Liu, ${ }^{2}$ \\ Yuanhua Liu, ${ }^{2}$ Ting Fu, ${ }^{2}$ Pei Hao, ${ }^{2}$ Yi Hao, ${ }^{4}$ Nan Shen, ${ }^{1}$ Chunde Bao, ${ }^{1}$ \\ Xiaoming Zhang ${ }^{\circ}$
}

\begin{abstract}
Handling editor Josef $S$ Smolen

- Additional material is published online only. To view please visit the journal online (http://dx.doi.org/10. 1136annrheumdis-2019215039).
\end{abstract}

For numbered affiliations see end of article.

\section{Correspondence to}

Professor Xiaoming Zhang, Key Laboratory of Molecular Virology \& Immunology, Institut Pasteur of Shanghai Chinese Academy of Sciences, Shanghai 200031, China; xmzhang@ips.ac.cn and Professor Chunde Bao, Department of Rheumatology, Renji Hospital, School of Medicine, Shanghai Jiao Tong University, Shanghai, China; baochunde_1678@126.com

CW, QF and QG contributed equally.

Received 9 January 2019 Revised 17 April 2019

Accepted 18 April 2019 Published Online First 29 May 2019
Check for updates

(c) Author(s) (or their employer(s)) 2019. Re-use permitted under CC BY-NC. No commercial re-use. See rights and permissions. Published by BMJ.

To cite: Wu C, Fu Q, Guo Q, et al. Ann Rheum Dis

2019;78:1090-1100.

\section{ABSTRACT}

Objectives A population of atypical memory B cells (AtMs) are greatly expanded in patients with active lupus, but their generation and pathophysiological roles are poorly defined. The aim of this study was to comprehensively characterise lupus AtMs with a purpose to identify therapeutic clues to target this B cell population in lupus.

Methods Peripheral B cell subsets were measured by flow cytometry. Sorting-purified B cell subsets were subject to RNA sequencing and functional studies. Plasma cytokines and secreted immunoglobulins were detected by Luminex or ELISA. In situ renal B cells were detected by multiplexed immunohistochemistry.

Results $\mathrm{CD} 24^{-} \mathrm{CD} 20^{\text {hi }}$ AtMs were strongly increased in two Chinese cohorts of patients with treatmentnaïve lupus. Gene expression profile indicated that B cell signalling and activation, lipid/saccharide metabolism and endocytosis pathways were abnormally upregulated in lupus AtMs. In addition, the mammalian target of rapamycin complex 1 (mTORC1) pathway was remarkably activated in lupus AtMs, and blocking mTORC1 signalling by rapamycin abolished the generation of T-bet ${ }^{+} B$ cells and terminal differentiation of lupus AtMs. Furthermore, lupus AtMs displayed a dysfunctional phenotype, underwent accelerated apoptosis, poorly co-stimulated T cells and produced proinflammatory cytokines. Interestingly, lupus AtMs were in a paradoxically differentiated status with markers pro and against terminal differentiation and enriched with antinucleosome reactivity. Finally, AtMs were accumulated in the kidneys of patients with lupus nephritis and associated with disease severity.

Conclusions These findings demonstrated that mTORC1-overactivated lupus AtMs are abnormally differentiated with metabolic and functional dysregulations. Inhibiting mTORC1 signalling might be an attractive option to target AtMs and to improve therapeutic effectiveness in patients with lupus.

\section{INTRODUCTION}

Systemic lupus erythematosus (SLE) is an autoimmune disease triggered by loss of self-tolerance and the resultant autoreactive cellular and humoral immune responses, leading to a striking heterogeneity of clinical manifestations and organ dysfunction. ${ }^{12}$ B cell abnormality plays a central

\section{Key messages}

What is already known about this subject?

- T-bet ${ }^{+}$CD $11 \mathrm{c}^{+}$atypical memory B cells (AtMs) are greatly expanded in lupus and implicated in the pathogenesis of systemic lupus erythematosus (SLE).

What does this study add?

- We find that the mTORC1 pathway is highly activated in lupus AtMs and plays a critical role in the generation and terminal differentiation of these cells. Importantly, inhibiting mTORC1 signalling by rapamycin blocks T-bet ${ }^{+} \mathrm{B}$ cell generation and the terminal differentiation of AtMs.

- Our study also demonstrates that AtMs are aberrantly differentiated, metabolically abnormal and functionally dysregulated. In addition, the abundance of AtMs both in the blood and in the kidneys of patients with lupus nephritis reflects the disease activity, indicating that AtMs could be a novel biomarker for patients with lupus.

How might this impact on clinical practice or future developments?

- Targeting AtMs by inhibiting mTORC1 signalling pathway could be an attractive option to improve therapeutic effectiveness in patients with SLE.

role in the development of SLE by contributing to the overproduction of autoantibodies, cytokines and augmented presentation of autoantigens to $\mathrm{T}$ cells. $^{34}$

The B cell compartment is highly distorted in patients with active SLE. Transitional B cells and plasmablasts are greatly increased, while non-switched memory B cells are decreased in active SLE. ${ }^{5-7}$ In addition, a population of atypical memory $B$ cells (AtMs) sharing similar phenotypes and identified as CD 19 hi 8 CD $19{ }^{\text {hi }} \mathrm{CD} 21^{\text {lo } 59},{ }^{5} \mathrm{CD}_{27}{ }^{-} \mathrm{IgD}^{-10} 11$ are expanded in active SLE. Increased frequency of these AtMs has been associated with high disease activity and disease-specific autoantibodies such as anti-Smith $(\mathrm{Sm})$ antibody, ${ }^{8} 10$ suggesting that these 
cells are associated with disease development. Phenotypically similar B cells were also expanded in other autoimmune diseases like primary Sjoegren's syndrome, systemic sclerosis ${ }^{5}$ and infectious diseases like HIV infection, ${ }^{12}$ hepatitis $\mathrm{C}$ virus infection ${ }^{13}$ and malaria. ${ }^{14}$ Thus, an environment of chronic inflammation seems to promote the generation of these B cells.

Recent studies have revealed that human AtMs specifically express transcription factor (TF) T-bet and integrin CD $11 \mathrm{c}^{15-17}$ which are closely linked to a population of $\mathrm{B}$ cells named age-associated $\mathrm{B}$ cells (ABCs) in aged female mice and young lupusprone mice. ${ }^{18} 19$ Murine ABCs are T-bet ${ }^{+} \mathrm{CD} 11 \mathrm{c}^{+}$and enriched with antichromatin autoantibodies which play an essential role in lupus development. ${ }^{16}{ }^{19}$ Furthermore, T-bet is necessary and sufficient for the generation of murine $\mathrm{CD} 11 \mathrm{c}^{+} \mathrm{ABCs}^{20}$ and deletion of T-bet in $\mathrm{B}$ cells causes a loss of $\mathrm{CD} 11 \mathrm{c}^{+} \mathrm{ABC}$ and the amelioration of disease in lupus-prone mice. ${ }^{16}{ }^{21}$ These studies demonstrated a common pathogenic role of T-bet ${ }^{+} \mathrm{CD} 11 \mathrm{c}^{+} \mathrm{B}$ cells in both human lupus and murine lupus models.

The identification of a population of atypical memory T-bet ${ }^{+} \mathrm{CD} 11 \mathrm{c}^{+} \mathrm{B}$ cells in patients with SLE raises the possibility that targeting this population may open new therapeutic opportunities for this complex disease, as the efficacies of B cell depletion therapy by targeting whole B cells in patients with SLE are highly variable. ${ }^{122}$ Particularly, it was reported that an expanded population of CD19 ${ }^{\text {hi }}$ AtMs in patients with SLE predicts a poor clinical response to rituximab treatment. ${ }^{8}$ Although several factors including Toll-like receptor (TLR) 7, interferon gamma (IFN- $\gamma$ ), interleukin (IL) 21 have been implicated in the differentiation of AtMs, ${ }^{19} 202324$ no clinically targetable pathway has been identified so far. Thus, to identify the key pathway leading to their generation and to develop feasible strategies to block their development represent an urgent need in lupus B cell study.

In this study, we aim to perform a comprehensive study of lupus AtMs at molecular, cellular, functional and clinical levels, with a purpose to uncover the unknown nature of AtMs and provide clues to target these cells in clinics.

\section{RESULTS}

AtMs are increased in patients with lupus and correlated with disease activity

We have analysed peripheral B cell subsets in a Chinese population of patients with new-onset treatment-naïve SLE by flow cytometry and observed that a population of $\mathrm{CD} 24^{-} \mathrm{CD} 20^{\text {hi }} \mathrm{B}$ cells was significantly increased in patients with SLE compared with patients with new-onset rheumatoid arthritis (RA) and healthy donors (HDs) (mean \pm SEM, 7.54\% $\pm 0.87 \%$, range $0.19 \%-$ $25.3 \% ; 3.07 \% \pm 0.45 \%$, range $0.67 \%-10.6 \% ; 1.56 \% \pm 0.15 \%$, range $0.39 \%-3.68 \%$; respectively) (figure 1A,B) (Detailed characteristics of the three groups were listed in online supplementary table 1). Phenotypical analysis revealed that the $\mathrm{CD} 24^{-} \mathrm{CD} 20^{\text {hi }} \mathrm{B}$ cells expressed high levels of CD11c, T-bet and memory marker CD95, but didn't express CD21, while the expression of the classical memory marker CD27 was variable (figure 1C), indicating that the $\mathrm{CD} 24^{-} \mathrm{CD} 20^{\text {hi }}$ B cells were corresponding to a population of AtMs identified in SLE ${ }^{101617}$ as well as in chronically infectious diseases ${ }^{12} 14$ and the healthy elderly. ${ }^{25}$

We then assessed the clinical relevance of these lupus AtMs. We found that the frequency of circulating AtMs was positively correlated with Systemic Lupus Erythematosus Disease Activity Index (SLEDAI) Scores, titres of serum antinucleosome antibody (ANUA) and anti-dsDNA, while negatively correlated with serum complement 3 (C3) and C4 (figure 1D and online supplementary figure $1 \mathrm{~A})$. We further showed that the frequency of
AtMs was much higher in lupus nephritis (LN) compared with those patients without renal involvement (figure 1E), and was negatively correlated with blood haemoglobin concentration (online supplementary figure 1B). Furthermore the frequency of lupus AtMs was positively correlated with a panel of proinflammatory cytokines including IL-6, IL-18, IFN- $\alpha$ and IFN- $\gamma$ in plasma (online supplementary figure 1C). We confirmed the correlation between AtMs and disease activity in a second cohort of 19 patients with new-onset lupus (online supplementary table 2). Either defined as $\mathrm{CD} 24^{-} \mathrm{CD} 20^{\mathrm{hi}}$ or $\mathrm{CD} 11 \mathrm{c}^{+} \mathrm{CD} 24^{-} \mathrm{CD} 20^{\mathrm{hi}}$, the frequencies of both AtMs were positively correlated with SLEDAI Scores and negatively correlated with serum C3 concentrations (online supplementary figure 1D-F).

Twenty-three patients from the first cohort who have received standard therapy were followed up for 24 weeks (online supplementary tables 3 and 4). The frequencies of AtMs were rapidly decreased 4 weeks after treatment and maintained at relatively low levels thereafter (figure 1F). Strikingly, we observed a significant increase of AtMs in a subgroup of eight patients who experienced relapses or showed resistance to the treatment at week 24 (figure 1G). These results suggest that AtMs could be a potential biomarker to monitor disease activity and predict disease flare.

\section{Lupus AtMs exhibit a unique gene expression profile with BCR activation and metabolic dysregulation}

To explore the molecular features of lupus AtMs, we performed an RNA-seq-based gene expression profiling of AtMs and classical memory B cells (CMs) from six patients with new-onset SLE, as well as CMs from five HDs. Principal component analysis separated the three memory B cell populations apart (figure 2A). Venn diagrams showed that 578 genes were significantly upregulated and 384 genes were significantly downregulated in lupus AtMs compared with lupus CMs and healthy CMs, respectively (figure 2B). Gene clustering analysis classified the differentially expressed genes into two major clusters: lupus AtMs high and low expressions (figure 2C). With gene ontology terms as the reference, gene set enrichment analysis (GSEA) ${ }^{26}$ identified three pathways were predominantly upregulated in lupus AtMs compared with CMs: B cell signalling and activation, lipid and saccharide metabolism and endocytosis (figure 2D,E and online supplementary figure $2 \mathrm{~A}$ ). By contrast, RNA/protein synthesis-related pathways were dampened in lupus AtMs (online supplementary figure $2 \mathrm{~B}$ ).

Consistent with the above pathway analysis, we observed that lupus AtMs displayed heightened B cell receptor (BCR) signalling (pSyk(Y348), pSyk(Y352), pBTK(Y551/511) and pPLC 2 2(Y759)) compared with lupus $\mathrm{CMs}$ at steady state (figure $2 \mathrm{~F}, \mathrm{G}$ ). However, following BCR cross-linking, lupus CMs rapidly upregulated phosphorylated BCR signalling molecules while limited increase was observed in AtMs (online supplementary figure 2C). This $\mathrm{BCR}$ responding feature is similar to that observed in the AtMs from malaria-infected patients ${ }^{27}$ which indicates that AtMs are not intrinsically highly responding to BCR stimulation.

\section{mTORC1 pathway is highly activated and implicated in the differentiation of AtMs}

The increased BCR signalling in lupus AtMs at steady state prompted us to check the downstream signalling pathways. GSEA and gene differential analysis showed that the PI3K pathway was mobilised in lupus AtMs (figure 2D, online supplementary figure 3). Strikingly, using the HALLMARK database, GSEA identified that mTORC1 pathway, a key target of the PI3K/Akt pathway, was highly activated in lupus 
A

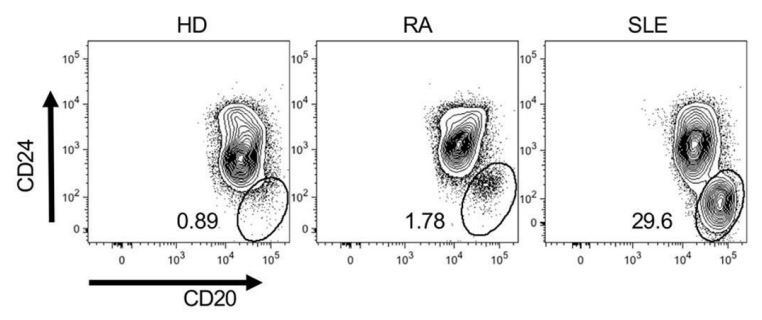

B

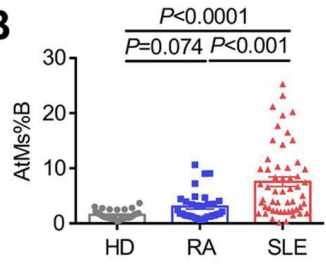

C
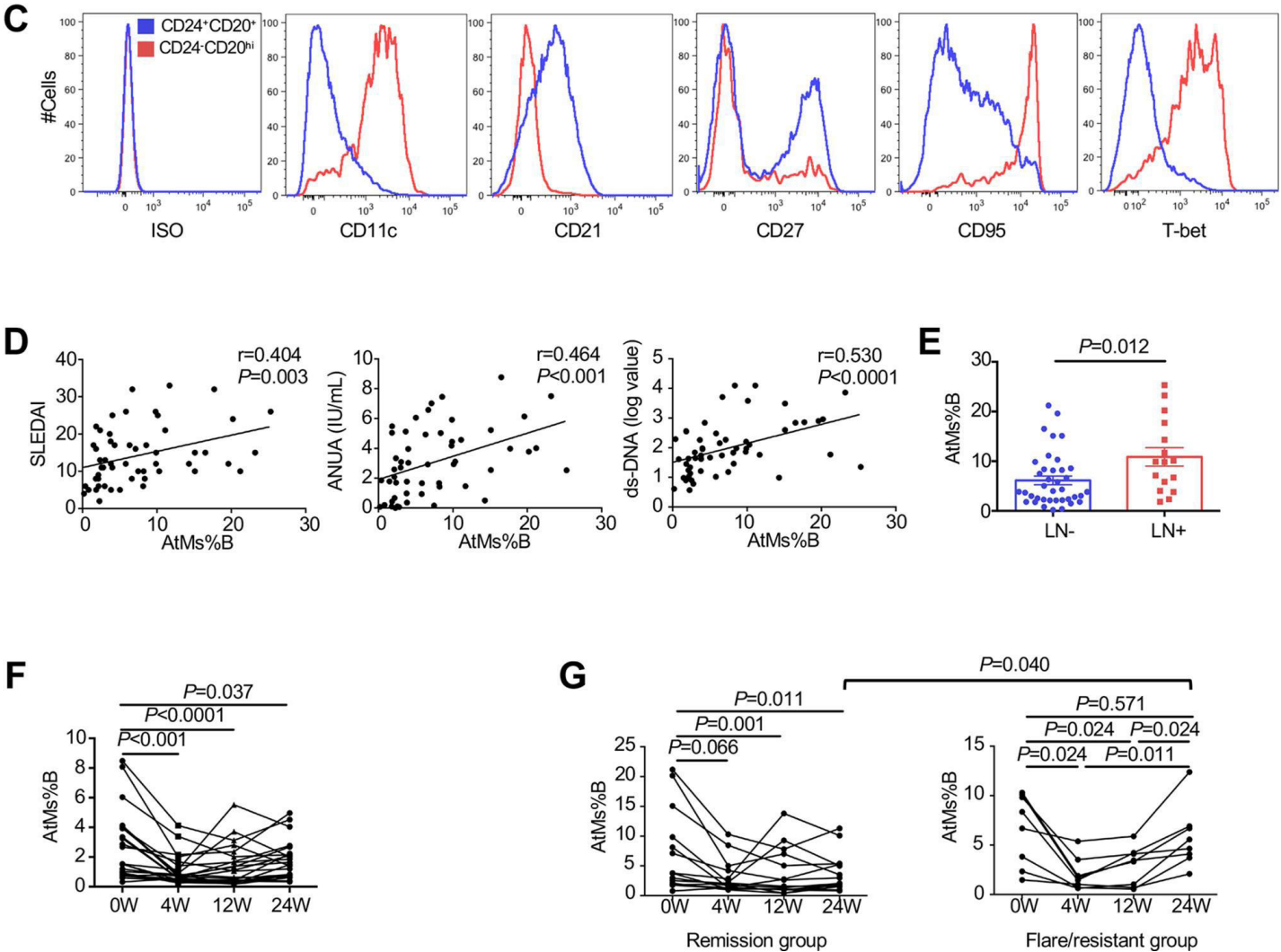

Figure 1 Atypical memory B cells (AtMs) were increased in the blood of patients with active SLE. (A) Flow cytometry analysis showed the frequency of circulating CD24-CD20 hi AtMs among total B cells (AtMs\%B) in healthy donor (HD), patient with RA and patient with new-onset SLE. (B) Comparison of circulating AtMs\%B among HD $(n=30)$, patient with RA $(n=33)$ and patient with new-onset SLE $(n=54)$. (C) Flow cytometry analysis of differentially expressed markers between peripheral $C D 24^{+} C D 20^{+} B$ cells and $C D 24^{-} C 20^{\text {hi }} B$ cells in patients with active SLE. For each marker, one representative plot out of three to four was shown. (D) Correlations between circulating AtMs\%B and SLEDAI Scores, titres of serum ANUA and antidsDNA of patients with new-onset SLE ( $n=54)$. (E) Comparison of circulating AtMs\%B between patients with new-onset lupus nephritis (LN) ( $n=16)$ and without $L N(n=38)$. (F) Changes of circulating AtMs\%B in patients with new-onset SLE before and after treatments (at weeks 0, 4, 12 and 24, $\mathrm{n}=23$ ). (G) Changes of circulating AtMs\%B in patients with new-onset SLE in the remission group $(n=15)$ and flare/resistant group ( $n=8)$ before and after treatments (at weeks $0,4,12$ and 24), and comparison of circulating AtMs\%B between two groups at week 24. Error bars indicated mean \pm SEM. $P$ values were determined by Kruskal-Wallis test with Dunn's multiple comparisons test (B), Spearman's rank correlation (D), Mann-Whitney test (E and $\mathrm{G}$ ) and Friedman test with Dunn's multiple comparisons test (F and G). ANUA, antinucleosome antibody; SLE, systemic lupus erythematosus.

AtMs compared with CMs (figure 3A). Consistently, we observed increased phosphorylations of mTOR(Ser2448) and S6(Ser235/236) in lupus AtMs, indicating activation of the mTORC1 pathway ${ }^{28} 29$ in these cells. By contrast, the phosphorylation level of Akt(Ser473), a marker of mTORC2 activation, ${ }^{28}$ didn't differ between AtMs and CMs in patients with SLE (figure 3B,C).

Next we investigated whether the mTORC1 pathway is involved in the differentiation of T-bet ${ }^{+}$AtMs. We chose an in vitro differentiation protocol by using IFN- $\gamma$ and a TLR7 agonist R848, as both IFN- $\gamma$ and TLR7 signalling were implicated in the differentiation of T-bet ${ }^{+}$AtMs. ${ }^{19} 2330$ The results showed that the combination of IFN- $\gamma$ and R848 had a synergistic effect on mTORC1 activation reflected by the increased frequency of p-S6 ${ }^{+}$(phosphorylated S6(Ser235/236)) cells and the generation of $\mathrm{CD} 11 \mathrm{c}^{+} \mathrm{T}_{-}$bet $^{+}$and CD11c T-bet ${ }^{+} \mathrm{B}$ cells

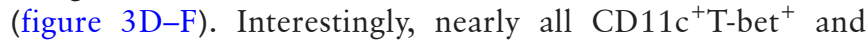
CD11c T-bet ${ }^{+} \mathrm{B}$ cells were $\mathrm{p}-\mathrm{S} 6^{+}$while the frequency of $\mathrm{p}-\mathrm{S} 6^{+}$ cells within $\mathrm{CD} 11 \mathrm{c}^{-} \mathrm{T}^{-}$bet $^{-} \mathrm{B}$ cells was significantly reduced (online supplementary figure $4 \mathrm{~A}, \mathrm{~B}$ ). The results suggest that T-bet expression in B cells could be associated with mTORC1 activation.

To further explore this connexion, we tested the effect of Rapamycin, a specific inhibitor of mTORC1, ${ }^{31}$ on the differentiation of T-bet ${ }^{+} \mathrm{B}$ cells. Consistent with an early report, ${ }^{32}$ Rapamycin decreased the viability and blocked the proliferation of IFN- $\gamma / \mathrm{R} 848$-stimulated B cells (online supplementary figure 4C-F). In gated live B cells, rapamycin 
A

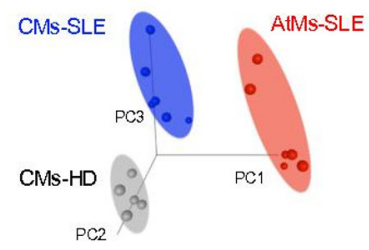

B

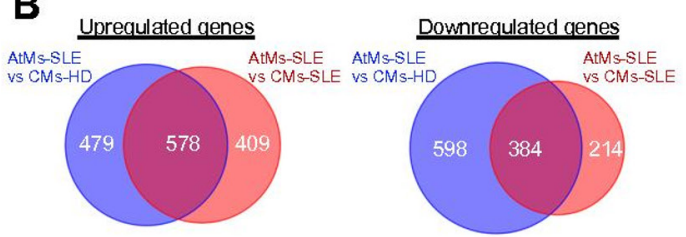

D

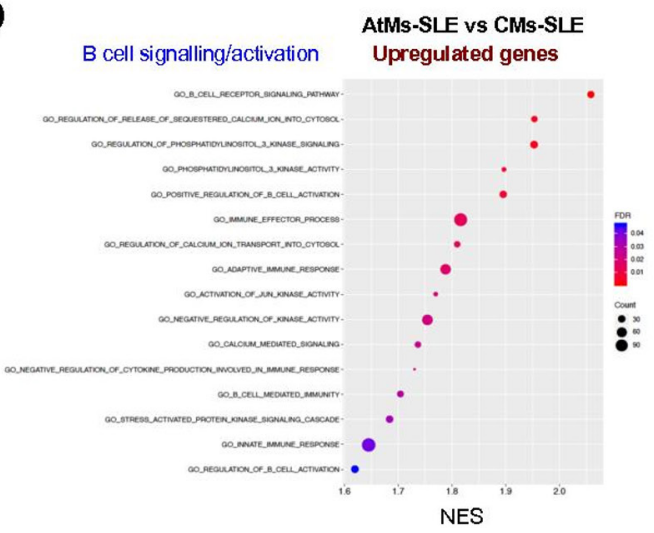

$\mathbf{F}$
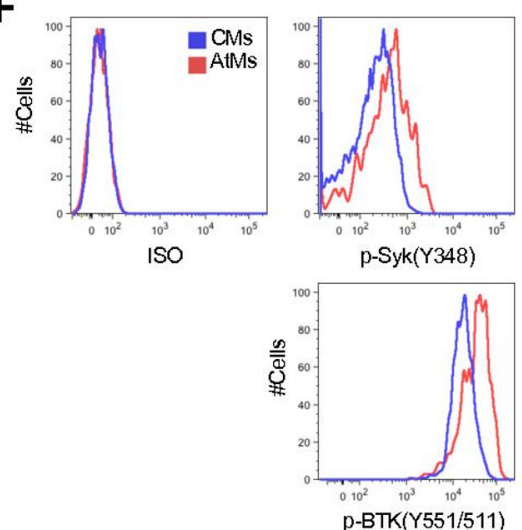
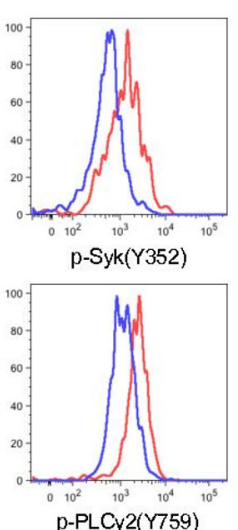

C

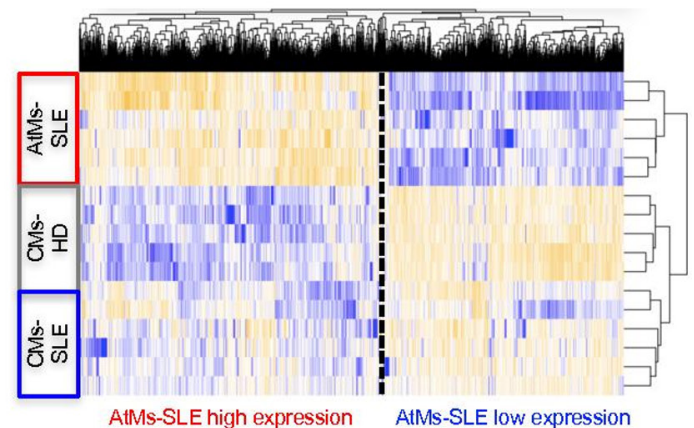

E Lipid and saccharide metabolism Upregulated genes

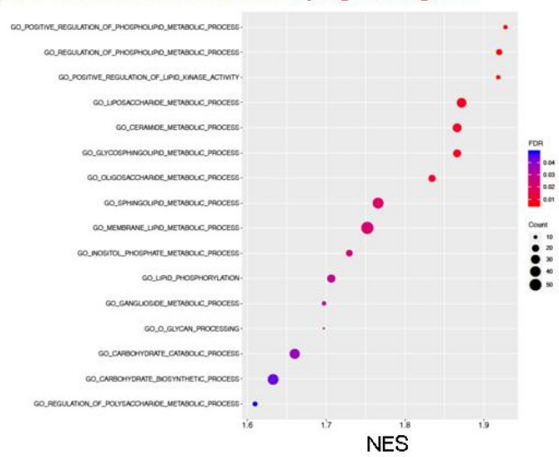

$\mathbf{G}$

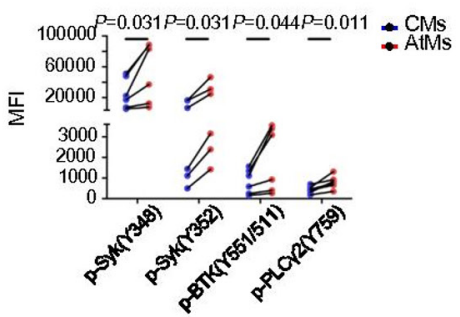

Figure 2 Lupus atypical memory B cells (AtMs) displayed a unique gene expression profile from CMs. (A) Principal component analysis (PCA) of the RNA-Seq data showed the differential gene expression profiles of CMs from HD ( $n=5)$, CMs and AtMs from patients with new-onset SLE $(n=6)$. (B) Venn diagrams of RNA-Seq differentially expressed genes (DEGs) of AtMs-SLE compared with CMs-SLE or CMs-HD. (C) Unbiased hierarchical clustering was performed to show the 2688 DEGs from AtMs-SLE, CMs-SLE and CMs-HD. The selection criterion was fold change (FC) $>2, P$ value adj $<0.05$ between any two populations. (D-E) Gene set enrichment analysis (GSEA) identified the pathways of $B$ cell signalling/activation (D) and lipid and saccharide metabolism (E) were highly enriched in AtMs-SLE compared with CMs-SLE based on gene expression data from RNA-seq. (F-G) Flow cytometry analysis (F) and accumulated data (G) of the differentially expressed markers of BCR signalling at baseline between AtMs and CMs from same patients with SLE $(\mathrm{n}=6)$. $P$ values were determined by paired $t$ test $(\mathrm{G})$. CMs, classical memory B cells; HD, healthy donor; NES, normalised enrichment score; PC1, Principal Component 1; PC2, Principal Component 2; PC3, Principal Component 3; ISO, isotype; SLE, systemic lupus erythematosus.

strongly decreased the frequencies of $\mathrm{p}-\mathrm{S} 6^{+}$, as well as CD $11 \mathrm{c}^{+} \mathrm{T}^{-}$bet $^{+}$and CD11 $\mathrm{c}^{-} \mathrm{T}^{-}$bet $^{+} \mathrm{B}$ cells (figure $3 \mathrm{E}$ ). Rapamycin also strongly inhibited T-bet expression at mRNA level in stimulated B cells (figure 3F). To differentiate whether the inhibition of mTORC1 activation and T-bet expression by rapamycin is due to the inhibition of proliferation, we focused on non-proliferating B cells. The results showed that the phosphorylation of S6(Ser235/236) and T-bet expression were not dependent on proliferation and rapamycin strongly decreased the frequencies of $\mathrm{p}-\mathrm{S} 6^{+}$and T-bet ${ }^{+} \mathrm{B}$ cells in gated live non-proliferating B cells (online supplementary figure $4 \mathrm{G}, \mathrm{H}$ ). Collectively, these studies reveal a potential link of mTORC1 activation and the differentiation of T-bet ${ }^{+} \mathrm{B}$ cells. 
A

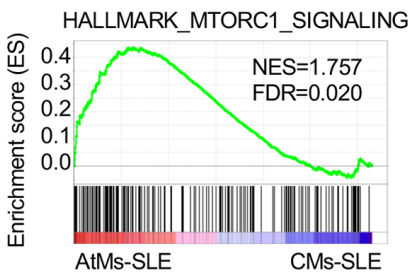

B
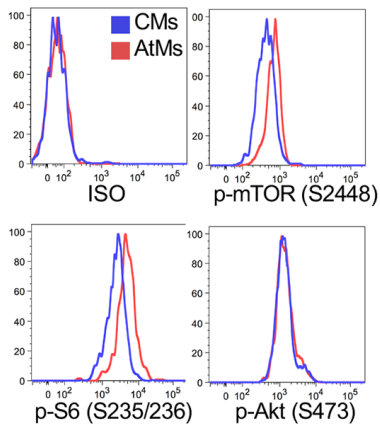

C

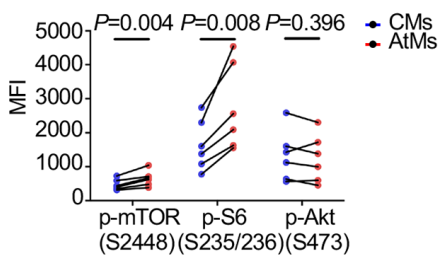

D
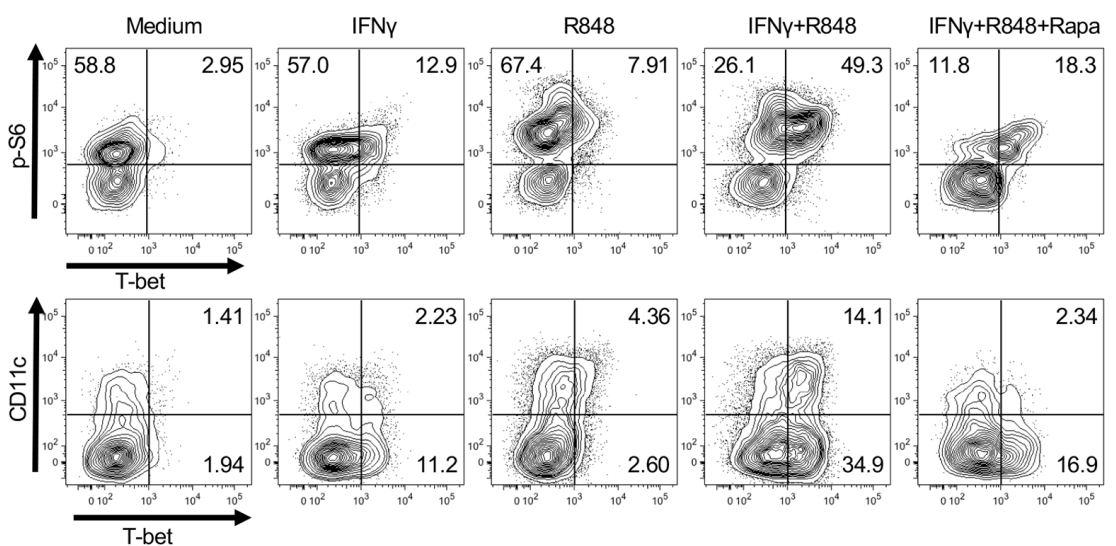

E
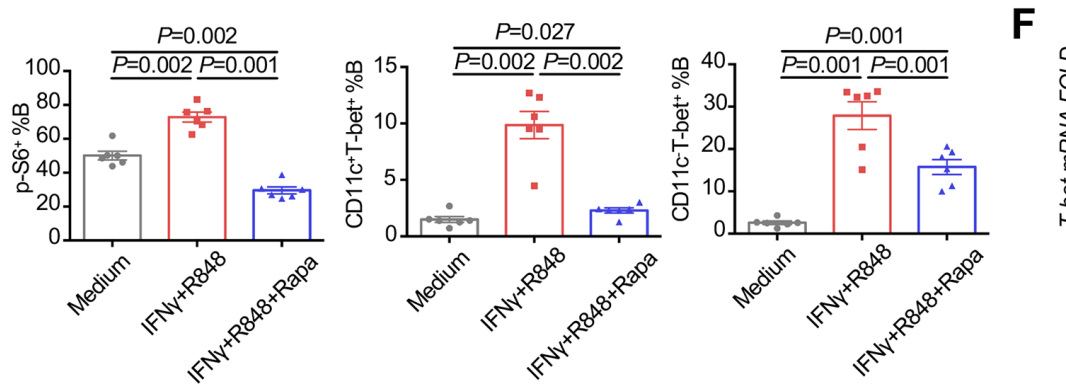

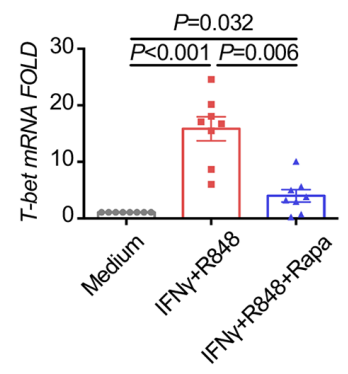

Figure 3 mTORC1 signalling pathway was implicated in the differentiation of atypical memory B cells (AtMs). (A) Gene set enrichment analysis (GSEA) identified that the mTORC1 signalling pathway was enriched in lupus AtMs compared with CMs. (B) Differential expression of mTOR signalling-related phosphorylated proteins between AtMs and CMs from the same patient with SLE, analysed by flow cytometry. One representative experiment out of four was shown. (C) Comparison of mean fluorescence intensities (MFI) of the mTOR signalling pathway-related phosphorylated proteins between AtMs and CMs from the same patients with SLE ( $n=6)$. (D-E) Representative flow cytometry plots (D) and accumulated data (E) of the frequencies of $p-S 6^{+}, C D 11 c^{-} T$-bet ${ }^{+}$and $C D 11 c^{+} T$-bet ${ }^{+} B$ cells generated from stimulated healthy donor $B$ cells $(n=6)$. For $D, B$ cells were stimulated with IFN $\gamma$ and R848 alone or in combination in the absence or presence of $0.1 \mathrm{nM}$ rapamycin (rapa). For $\mathrm{E}, \mathrm{B}$ cells were stimulated with the combination of IFN $\gamma$ and R848 in the absence or presence of $0.1 \mathrm{nM}$ rapamycin. (F) T-bet mRNA levels were measured in stimulated B cells for 6 hours $(\mathrm{n}=8)$. The stimulation conditions were the same as in $(\mathrm{E})$ and the concentration of rapamycin was $10 \mathrm{nM}$. Error bars indicated mean $\pm S E M$. $P$ values were determined by paired $t$ test (C) and one-way analysis of variance (ANOVA) with Holm-Sidak's multiple comparisons test (E and F). CMs, classical memory B cells; NES, normalised enrichment score; FDR, false discovery rate; ISO, isotype; mTOR, the mammalian target of rapamycin; SLE, systemic lupus erythematosus.

\section{Lupus AtMs are enriched with antinucleosome reactivity despite impaired terminal differentiation}

There have been inconsistent reports for the terminal differentiation potentials of AtMs, with evidence of normal ${ }^{17}{ }^{33}$ or resistant ${ }^{12} 2734$ to plasma cell differentiation. Our RNA-seq data showed that lupus AtMs expressed higher levels of B cell identity genes including PAX5, BCL6 and AICDA, and higher amounts of plasma cell-related genes like IRF4, XBP1 and SLAMF7 (figure 4A). At the protein level, AtMs expressed high levels of PAX5, BCL6 and IRF4 (figure 4B). PAX5 and BCL6 are TFs to maintain B cell identity while IRF4 is considered to promote plasma cell differentiation, ${ }^{35}$ thus AtMs seem to be in an aberrant state of differentiation.

Then we tested the terminal differentiation capacity of sorted lupus AtMs and CMs with a strong stimulating protocol (cytosine-phosphate-guanidine (CpG) 2006/IL-2/IL-10) for 7 days. We found that lupus AtMs displayed a compromised capacity to differentiate into $\mathrm{CD} 27^{\mathrm{hi}} \mathrm{CD} 38^{\text {hi }}$ plasmablasts (figure $4 \mathrm{C}, \mathrm{D}$ ) and to secrete immunoglobulins compared with CMs (figure 4E). Interestingly, after normalisation to the same concentrations, the 
A

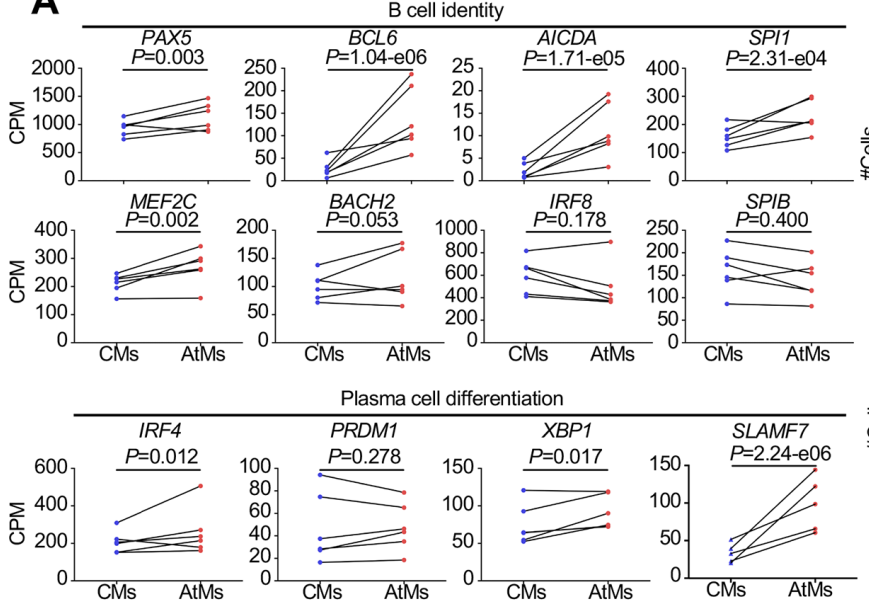

B
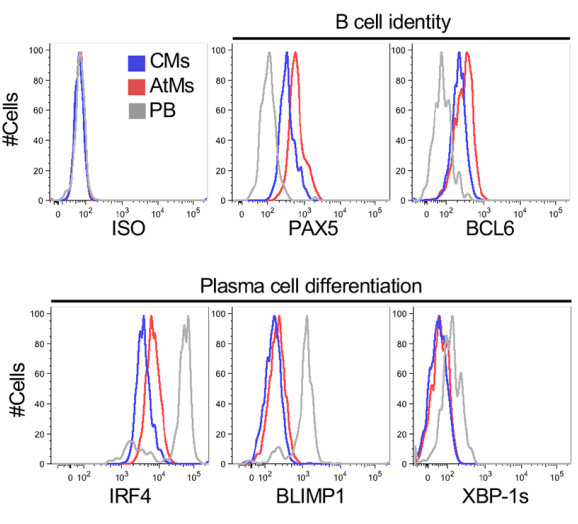

$\mathbf{E}$
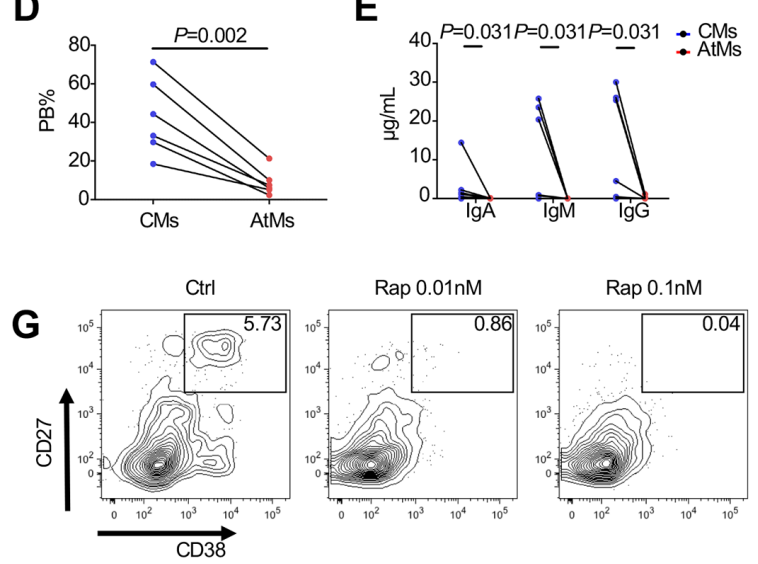

H
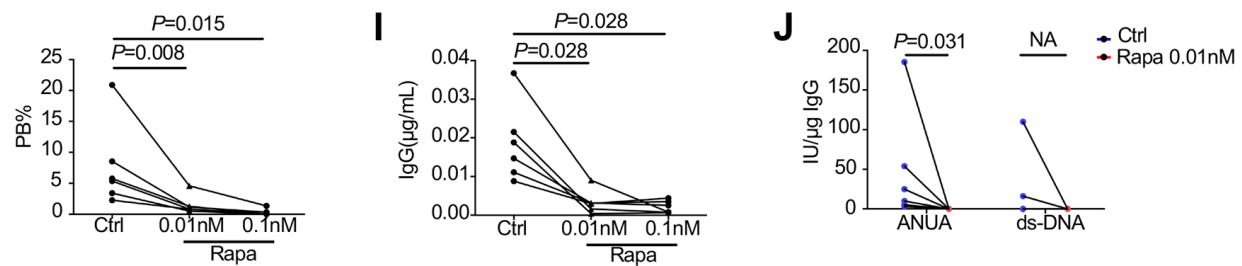

Figure 4 AtMs exhibited altered ability of antibody secretion. (A) RNA-Seq data were used to compare the expression profiles of B cell identity and plasma cell differentiation-related genes between AtMs and CMs from active patients with $S L E(n=6)$. (B) Flow cytometry analysis to show $B$ cells and plasma cell differentiation-related transcription factors between CMs and AtMs from the same patients with SLE. One representative experiment out of four was shown. (C-D) Representative flow cytometry plots (C) and accumulated data ( $n=6)$ (D) to show the capacity of lupus AtMs and CMs to differentiate into $\mathrm{CD} 27^{\text {hi }} \mathrm{CD} 38^{\text {hi }}$ plasmablasts (PB) following the stimulation of $\mathrm{CpG}+\mathrm{IL}-2+\mathrm{IL}-10$ for 7 days. Shown were the frequencies among live cells. (E-F) Comparison of the concentrations of secreted immunoglobulin IgA, IgM and $\lg G(E)$ or ANUA and anti-dsDNA levels based on the normalised IgG concentration (F) between AtMs and CMs from patients with SLE ( $n=6)$. (G-H) Representative flow cytometry plots (G) and accumulated data $(H)(n=6)$ to show the capacity of lupus AtMs to differentiate into $C D 27^{\text {hi }} C D 38^{\text {hi }}$ plasmablasts following the stimulation of $\mathrm{CpG}$ $+\mathrm{IL}-2+\mathrm{IL}-10$ in the absence or presence of $0.01 \mathrm{nM}$ and $0.1 \mathrm{nM}$ rapamycin (rapa) for 7 days. (I-J) Changes in the concentration of IgG (I), ANUA and anti-dsDNA levels (J) secreted by AtMs-SLE after adding rapamycin $(n=6)$. For $A$, Limma+voom (paired samples test) was used for differential analysis and $P$ values were adjusted by the Benjamini-Hochberg multiple test. $P$ values were determined by paired $t$ test (D), Wilcoxon signed-rank test $(\mathrm{E}, \mathrm{F}, \mathrm{J})$, one-way analysis of variance (ANOVA) with Holm-Sidak's multiple comparisons test (H), and Friedman test with Dunn's multiple comparisons test (I). ANUA, antinucleosome antibody; AtMs, atypical memory B cells; CMs, classical memory B cells; ISO, isotype; SLE, systemic lupus erythematosus.

secreted IgG from AtMs showed an enrichment of ANUA, but not anti-dsDNA antibodies compared with that of CMs (figure 4F). It was reported that mTORC1 signalling was important for plasma cell differentiation and antibody secretion; $;{ }^{32} 36$ we found that rapamycin significantly blocked plasmablast generation, IgG and ANUA production from stimulated AtMs (figure 4G-J). Thus, the above studies indicate that lupus AtMs represented a unique $\mathrm{B}$ cell population aberrantly differentiated and enriched with antinucleosome reactivity. Furthermore, inhibiting the mTORC1 pathway could effectively block their terminal differentiation and the capacity to secrete autoantibodies.

Lupus AtMs are molecularly and phenotypically dysfunctional We observed a panel of genes encoding inhibitory receptors (eg, PDCD1, FCRL3, ILIRB1, ILIRB2) linked to dysfunction 
or exhaustion in $\mathrm{T}$ cells ${ }^{12} 27$ were highly expressed in lupus AtMs (online supplementary figure 5A), and the dysfunctional phenotype was confirmed at the protein levels for most markers detected (online supplementary figure 5B). Furthermore, markers of B cell development and activation also showed aberrations at molecular and protein levels (online supplementary figure $5 \mathrm{~A}$ and 6 ).

TFs play a decisive role in coordinating cell fate and functions, however, the TF network of AtMs is not clear. We found that lupus AtMs expressed a similar TF gene profile to dysfunctional $\mathrm{T}$ cells, ${ }^{37} 38$ including increased expression of BATF, FOXO1, IKZF2, NFATc2, MAF and decreased expression of MAML2, ZEB2, ZBTB20 and TCF7 (online supplementary figure $5 \mathrm{C}$ ). The enhanced expressions of NFATc2, BATF, FOXO1 and HELIOS were confirmed by flow cytometry (online supplementary figure 5B). Interestingly, we also found that lupus AtMs expressed higher levels of HIF1 $\alpha$ and c-Myc (online supplementary figure 5B), two TFs associated with T cell dysfunction ${ }^{39} 40$ and metabolism. ${ }^{4142}$ Thus, the dysfunctional phenotype of lupus AtMs could be linked to a unique metabolic status.

\section{Lupus AtMs are apoptosis-prone, defective for proinflammatory cytokine production and poorly co-stimulate T cells to proliferate}

Next we investigated the survival capacity of lupus AtMs. GSEA identified an apoptotic gene signature for lupus AtMs (figure 5A) and several antiapoptotic genes BCL2, PIM1, PIM2 and PIM3 were all decreased in AtMs compared with CMs (online supplementary figure 7A). The downexpression of BCL2 was confirmed at the protein level in AtMs by flow cytometry (online supplementary figure $7 \mathrm{~B}$ ). Consistent with these data, we found that the sorted lupus AtMs displayed significantly less survival advantage and higher frequency of active caspase-3-positive cells than CMs following overnight culture (figure 5B,C).

The RNA-seq data showed that lupus AtMs expressed lower proinflammatory molecules IL6 and Tumor Necrosis Factor (TNF) and higher anti-inflammatory molecules IL10 and Transforming Growth Factor Beta-1 (TGFB1) (online supplementary figure $7 \mathrm{C}$ ). We found that lupus AtMs showed much reduced capacity to produce proinflammatory cytokine IL- 6 and TNF- $\alpha$ following polyclonal stimulations (figure $5 \mathrm{D}$ and online supplementary figure 7D).

B cells also play an active role in co-stimulating $\mathrm{T}$ cells. ${ }^{4}$ Compared with lupus CMs, AtMs exhibited a significantly decreased capacity to co-stimulate $\mathrm{CD}^{+}{ }^{+} \mathrm{T}$ cells to proliferate, as checked by carboxyfluorescein succinimidyl ester (CFSE) dilution (figure 5E), which was accompanied with a diminished survival of AtMs (figure 5F). These results suggest that lupus AtMs are apoptosis-prone, defective for proinflammatory cytokine production and poorly provide co-stimulatory signals to $\mathrm{T}$ cells under the current experimental conditions.

\section{AtMs are heavily infiltrated into the kidneys of patients with LN}

Finally we performed an in situ study of AtMs in renal tissues of patients with LN by multiplexed immunohistochemistry (figure $6 \mathrm{~A}$ and online supplementary figure $8 \mathrm{~A}$ ). The renal tissues from patients with LN (detailed characteristics are in online supplementary table 5) showed a tendency of increased infiltration of $\mathrm{CD} 20^{+} \mathrm{B}$ cells compared with controls (online supplementary figure $8 \mathrm{~B}$ ). Interestingly, CD $20^{+} \mathrm{T}^{- \text {bet }^{+}}$AtMs were abundantly detected in the renal tissues of patients with LN compared with controls for both frequency and density (figure 6B). Consistent with the data from peripheral AtMs, we observed that $\mathrm{T}^{- \text {bet }^{+}}$AtMs from renal tissues of patients with LN displayed significantly higher levels of phosphorylated S6(Ser235/236) than T-bet B cells (figure 6C,D).

The frequency of AtMs in renal tissues was positively correlated with that in peripheral blood (figure 6E), suggesting that peripheral AtMs frequency may predict the degree of renal AtMs infiltrations. Furthermore, the frequency of renal AtMs was significantly higher in a group with high renal SLEDAI Scores $(12 \sim 16)$ than the group with low renal SLEDAI Scores $(0 \sim 4)$ (figure $6 \mathrm{~F})$. When different pathological types were taken into consideration, type IV LN showed a significantly higher frequency of renal AtMs than that of type III+V (figure 6G). Moreover, the density of renal AtMs was positively correlated with SLEDAI Scores, serum anti-dsDNA titres, urine proteins and renal function indicators while negatively correlated with serum C3 and albumin levels (online supplementary figure $8 \mathrm{C}, \mathrm{D})$. These results clearly demonstrated that the degree of renal AtM infiltration is closely associated with disease activity and renal dysfunction, hinting that infiltrated AtMs may directly play a pathogenic role in situ.

\section{DISCUSSION}

The identification of AtMs with a phenotype of CD $19^{\text {hi }}$ CD $21^{\text {lo/- }}$ in lupus can be dated back to $2002 .{ }^{9}$ Since then, a series of studies have reported phenotypically similar B cells to be expanded in patients with lupus. ${ }^{5-11} 172333$ By integrating phenotypical, molecular, functional and histological approaches, the current study adds new evidence that AtMs are abnormally differentiated with metabolic and functional dysregulation, and associated with disease activity. Particularly, we have identified that the mTORC1 pathway is highly activated in lupus AtMs and potentially involved in their generation and terminal differentiation.

In mice, it has been shown that ABCs (murine AtMs) arose from follicular B cells ${ }^{18}$ and a lot of factors contributed to their generation, including TLR7, IFN- $\gamma$, BCR, IL-21, CD40 and major histocompatibility complex (MHC) II molecules. ${ }^{19} 202324$ Similarly, studies in humans also implicated TLR7/TLR9, IFN- $\gamma$ and IL-21 in the generation of T-bet ${ }^{+}$AtMs-like B cells. ${ }^{1723} 33$ However, to our understanding, the key signalling pathway leading to their generation has not been unveiled. Interestingly, we identified that the mTORC1 pathway was particularly elevated in human lupus AtMs and blocking this pathway inhibited the differentiation of T-bet ${ }^{+}$AtMs-like B cells. Furthermore, the mTORC1 pathway is known to be critical for plasma cell differentiation and antibody production. ${ }^{43}$ We confirmed this point by showing that rapamycin strongly decreased the antibody secretion from stimulated lupus AtMs. Given the promising results from a few clinical trials to use rapamycin (sirolimus) to treat patients with lupus, ${ }^{44}$ our current study hints that some effect of rapamycin could be via inhibiting the generation and terminal differentiation of AtMs.

AtMs expressing a panel of dysfunctional/inhibitory markers have been reported in $\mathrm{HIV}^{12}$ and malaria ${ }^{27}$ infections, as well as in patients with lupus. ${ }^{1733}$ We extended these studies by demonstrating that lupus AtMs expressed dysfunctional markers, and shared a TF profile similar to dysfunctional T cells. ${ }^{37}{ }^{38}$ Interestingly, lupus AtMs also upregulate the two metabolism-related TFs HIF1 $\alpha$ and c-Myc, ${ }^{41}{ }^{42}$ which may be linked with the upstream mTORC1 activation and general metabolic aberrations, raising an important question to be addressed in future study.

There is some inconsistence about the capacity of AtMs to undergo terminal differentiation. Several studies have shown 

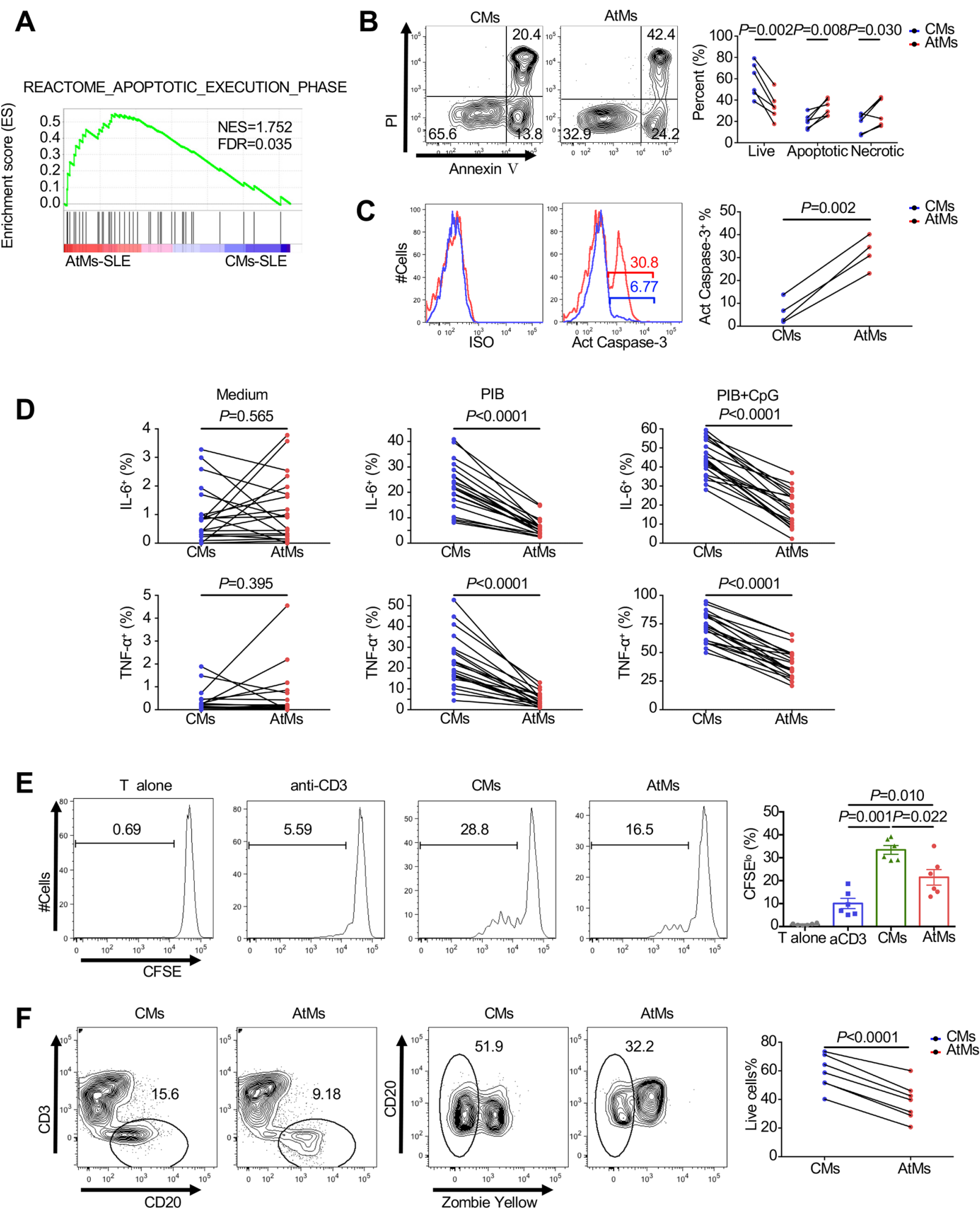

Figure 5 AtMs exhibited increased apoptosis potential, defective for proinflammatory cytokine production and impaired ability to co-stimulate T cells. (A) Gene set enrichment analysis (GSEA) indicated that the apoptotic execution phase pathway was enriched in lupus AtMs compared with CMs. (B) Representative flow cytometry plots and accumulated data showed the survival, apoptosis and necrosis rates of lupus AtMs and CMs cultured for 18-20 hours in vitro $(n=6)$. (C) Flow cytometry analysis and accumulated data to show the differentially expressed active caspase-3 between AtMs and CMs from the same patients with SLE after incubated at $37^{\circ} \mathrm{C}$ for 20 hours ( $\left.n=4\right)$. (D) Accumulated data showed the capacity of AtMs and CMs from active patients with SLE $(n=20)$ to produce IL- 6 and TNF- $\alpha$. PBMCs were stimulated with PIB or PIB plus CpG for 5 hours and then the cytokines were detected by flow cytometry. (E) Representative flow cytometry plots (left) and accumulated data (right) showed the ability of AtMs and CMs to co-stimulate $\mathrm{CD}^{+} \mathrm{T}$ cells to proliferate in vitro ( $\left.\mathrm{n}=6\right)$. (F) Representative flow cytometry plots (left) and accumulated data (right) to show the survival rate of CMs and AtMs co-clutured with $C D 4^{+} T$ cells $(n=6)$. Error bars indicated mean \pm SEM. $P$ values were determined by paired $t$ test $(B-D, F)$ and one-way analysis of variance (ANOVA) with Holm-Sidak's multiple comparisons test (E). AtMs, atypical memory B cells; CMs, classical memory B cells; NES, normalised enrichment score; FDR, false discovery rate; PI, Propidium lodide; ISO, isotype; PIB, PMA+lonomycin+Brefeldin A; CpG, cytosinephosphate-guanidine; CFSE, carboxyfluorescein succinimidyl ester; PBMCs, peripheral blood mononuclear cells; SLE, systemic lupus erythematosus. 
A
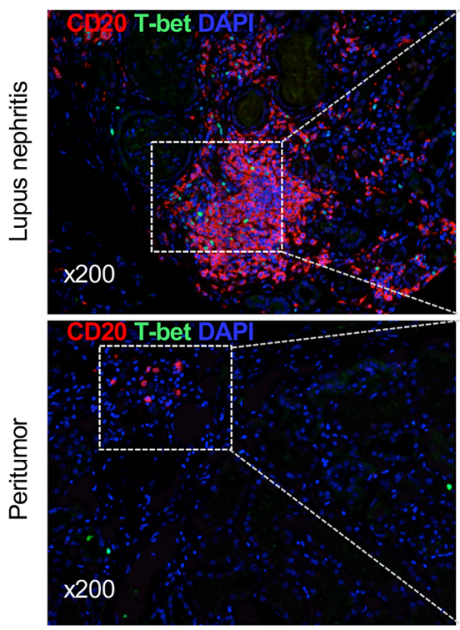

C

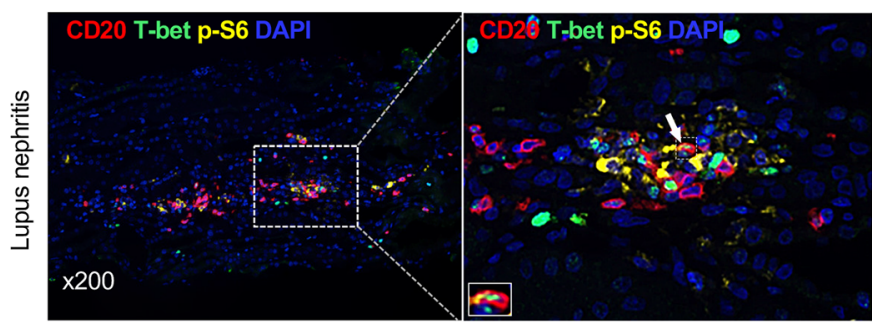

E

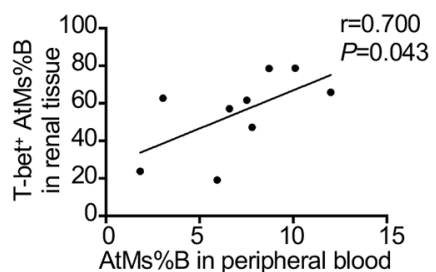

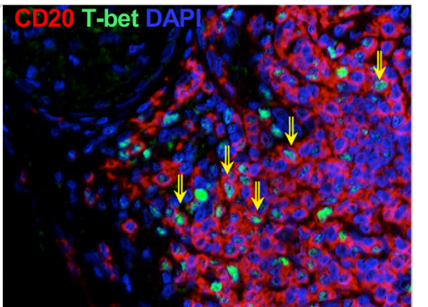

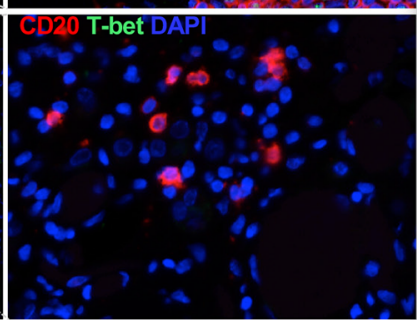

$\mathbf{F}$

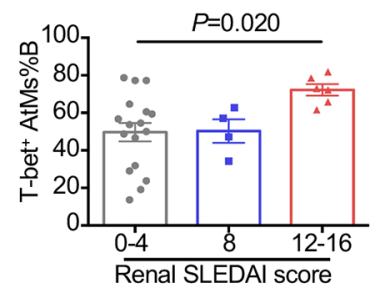

B
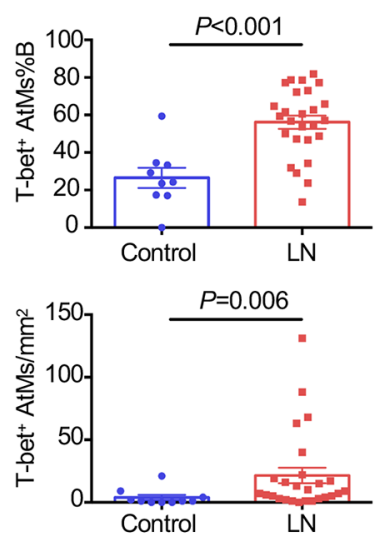

D

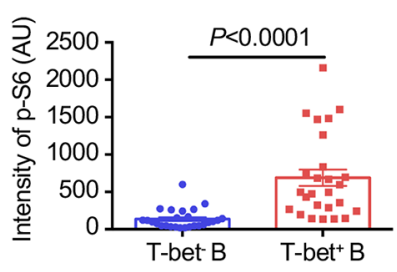

$\mathbf{G}$

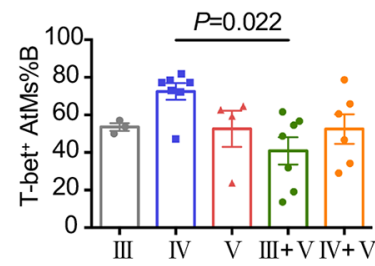

Figure $6 \mathrm{~T}_{\text {bet }}^{+}$AtMs infiltrated into the renal tissues of patients with lupus nephritis (LN). (A) Multiplexed IHC was applied to detect the CD20 ${ }^{+} \mathrm{T}$ bet $^{+}$AtMs in renal tissues of patients with LN. Peritumor renal tissue was used as control. (B) Comparison of the frequency (T-bet ${ }^{+}$AtMs $\% B$ ) and density of T-bet ${ }^{+}$AtMs in renal tissues of $L N(n=27)$ and peritumor renal tissues $(n=10)$. (C) T-bet ${ }^{+} B$ cells showed higher expression of $p$ - 56 than T-bet $B$ cells in renal tissues of patients with LN. (D) Comparison of the fluorescent intensity of p-S6 in 26 T-bet and 26 T-bet $^{+}$B cells in renal tissues from three patients with LN. (E) Correlation between T-bet ${ }^{+} A t M s \% B$ in renal tissues and AtMs\%B in peripheral blood from the same patients with $L N$ $(n=9)$. (F) Patients with LN were divided into three groups based on renal SLEDAI Scores and the frequency of renal T-bet ${ }^{+}$AtMs was compared among the three groups ( $n=17,10,6$, respectively). (G) Comparison of the frequency of renal T-bet ${ }^{+}$AtMs among different pathological types of patients with LN (type III $(n=3)$, type IV $(n=7)$, type V $(n=4)$, type III $+V(n=7)$ and type IV $+V(n=6))$. Error bars indicated mean \pm SEM. $P$ values were determined by Mann-Whitney test (B and D), Spearman's rank correlation (E) and Kruskal-Wallis test with Dunn's multiple comparisons test (F and $G)$. AtMs, atypical memory B cells; AtMs\%B, frequency of AtMs among total B cells; AU, arbitrary unit; IHC, immunohistochemistry.

the compromised capacity of AtMs to undergo terminal differenation ${ }^{12} 2734$ and we obtained similar results. By contrast, in one lupus study, following the stimulation of TLR7/IL-21/IL-10, although lupus AtMs did show a proliferation defect compared with CMs, they produced comparable amount of $\operatorname{IgG}$ on a per cell basis. ${ }^{33}$ Using activated $\mathrm{T}$ cells as the stimulus, Wang et al found similar frequencies of antibody-secreting cells and $\mathrm{IgG}$ secretion from lupus AtMs and CMs. ${ }^{17}$ While the difference from the above studies can be ascribed to the different experimental conditions, it should be mentioned that lupus AtMs are in a paradoxically differentiated status: AtMs express high levels of both B cell identity genes SPI1, MEF2C, PAX5 and BCL6, as well as plasma cell-driving genes IRF4, XBP1 and SLAMF7. Our tentative explanation is that AtMs are in fact dragged by two opposing differentiation forces. Most likely AtMs are not essentially poised to plasma cell differentiation themselves, however, they may be efficiently mobilised into plasma cell pool under certain pathogenic conditions like lupus flare.

Our and other studies have shown that lupus AtMs are highly enriched with antinuclear autoantibodies, including Sm, Nucleosome and Chromatin. ${ }^{8161719}$ It's interesting to ask how these pathogenic antibodies are generated and diversified in vivo. The isotype non-switched lupus AtMs (named activated naive B cells) showed higher IgM mutation rates than those of naïve B cells, ${ }^{46}$ indicating that a mechanism of antibody mutation is working for non-switched AtMs. For switched AtMs, the IgG mutation rates were lower than those of switched memory B cells from the same patients with lupus. ${ }^{33}$ Together with lower expressions of CXCR4 and CXCR5 on AtMs, the above evidence suggests that AtMs are a population of extrafollicular B cells independent of 
germinal centre origin. Intriguingly, we found that lupus AtMs express high levels of AICDA, which encodes activation-induced cytidine deaminase (AID), a molecule critical for somatic mutation and immunoglobulin isotype switching. ${ }^{47}$ We propose that AtMs are uniquely differentiated from a population of self-reactive naïve $\mathrm{B}$ cells ${ }^{48}$ in the context of chronic stimulation. High expression of PAX5 and BCL6 will ensure B cell identity in order for AID to perform its function and generate autoantibodies with sufficient affinity. In the context of disease flare, lupus AtMs could be abundantly expanded and mobilised into plasma cells to produce high levels of pathogenic autoantibodies.

In summary, the current study reveals new molecular and functional features of lupus AtMs. Particularly, as mTORC1 is required for both the generation and the terminal differentiation of AtMs, targeting mTORC1 pathway may represent an alternative strategy to the current lupus therapies, especially for those patients refractory to conventional regimens.

\section{METHODS}

Details of methods are available in online supplementary materials section.

\section{Author affiliations}

'Department of Rheumatology, Renji Hospital, School of Medicine, Shanghai Jiao Tong University, Shanghai, China

${ }^{2}$ Key Laboratory of Molecular Virology \& Immunology, Institut Pasteur of Shanghai, Chinese Academy of Sciences, Shanghai, China

${ }^{3}$ Department of Laboratory Medicine, The First Hospital of Nantong, Nantong, China ${ }^{4}$ Department of Pathogen Biology, School of Basic Medicine, Tongji Medical College, Huazhong University of Science and Technology, Wuhan, China

Acknowledgements The authors thank Min Dai, Wei Fan, Fang Du, Chunyan Zhang, Huihua Ding, Qingran Yan, Yan Ye, Suli Wang, Xia Lv and Xinyue Lian for clinical support (Renji Hospital, School of Medicine, Shanghai Jiao Tong University, China), and Huihui Xu, Meiying Zhang and Yi Chen for technical support (Institut Pasteur of Shanghai, Chinese Academy of Sciences, China).

Contributors CW and SG performed experiments and analysed data. SS, ML, TF and TL performed experiments. QF, QG, SC, FC, ZC, YL and PH analysed data. XC and YH provided reagents. QG, NS, CB and XZ designed the research. CW, QF and XZ wrote the manuscript.

Funding This work was supported by the Strategic Priority Research Program (No. XDB29030302), Interdisciplinary Innovation Team, Frontier Science Key Research Project (No. QYZDB-SSW-SMC036), Chinese Academy of Sciences; 973 Program (No. 2014CB541904), the National Natural Science Foundation of China (Nos. 31470879, $31770960,81471596,8157575,81771733,81771737$ and 81861138010) and Nantong Science and Technology Programme (No. MS120170105).

Competing interests None declared.

Patient consent for publication Not required.

Provenance and peer review Not commissioned; externally peer reviewed.

Data availability statement Data are available in a public, open access repository. All data relevant to the study are included in the article or uploaded as supplementary information.

Open access This is an open access article distributed in accordance with the Creative Commons Attribution Non Commercial (CC BY-NC 4.0) license, which permits others to distribute, remix, adapt, build upon this work non-commercially, and license their derivative works on different terms, provided the original work is properly cited, appropriate credit is given, any changes made indicated, and the use is non-commercial. See: http://creativecommons.org/licenses/by-nc/4.0/.

\section{REFERENCES}

1 Kaul A, Gordon C, Crow MK, et al. Systemic lupus erythematosus. Nat Rev Dis Primers 2016;2.

2 Tsokos GC. Systemic lupus erythematosus. N Engl J Med 2011;365:2110-21.

3 Dörner T, Giesecke C, Lipsky PE. Mechanisms of B cell autoimmunity in SLE. Arthritis Res Ther 2011;13.

4 Dörner T, Lipsky PE. Beyond pan-B-cell-directed therapy - new avenues and insights into the pathogenesis of SLE. Nat Rev Rheumato/ 2016;12:645-57.

5 Wehr C, Eibel H, Masilamani M, et al. A new CD21low B cell population in the peripheral blood of patients with SLE. Clin Immunol 2004;113:161-71.
6 Rodríguez-Bayona B, Ramos-Amaya A, Pérez-Venegas JJ, et al. Decreased frequency and activated phenotype of blood CD27 IgD IgM B lymphocytes is a permanent abnormality in systemic lupus erythematosus patients. Arthritis Res Ther 2010;12.

7 Liu M, Guo Q, Wu C, et al. Type I interferons promote the survival and proinflammatory properties of transitional B cells in systemic lupus erythematosus patients. Cell Mol Immunol 2019:16:367-79.

8 Nicholas MW, Dooley MA, Hogan SL, et al. A novel subset of memory B cells is enriched in autoreactivity and correlates with adverse outcomes in SLE. Clin Immunol 2008;126:189-201

9 Warnatz K, Wehr C, Dräger R, et al. Expansion of CD19(hi)CD21(lo/neg) B cells in common variable immunodeficiency (CVID) patients with autoimmune cytopenia. Immunobiology 2002:206:502-13.

10 Wei C, Anolik J, Cappione A, et al. A new population of cells lacking expression of CD27 represents a notable component of the $B$ cell memory compartment in systemic lupus erythematosus. J Immunol 2007;178:6624-33.

11 Jacobi AM, Reiter K, Mackay M, et al. Activated memory B cell subsets correlate with disease activity in systemic lupus erythematosus: delineation by expression of CD27, IgD, and CD95. Arthritis Rheum 2008;58:1762-73.

12 Moir S, Ho J, Malaspina A, et al. Evidence for HIV-associated B cell exhaustion in a dysfunctional memory B cell compartment in HIV-infected viremic individuals. J Exp Med 2008:205:1797-805.

13 Charles ED, Brunetti C, Marukian S, et al. Clonal B cells in patients with hepatitis C virus-associated mixed cryoglobulinemia contain an expanded anergic CD21 low B-cell subset. Blood 2011;117:5425-37.

14 Weiss GE, Crompton PD, Li S, et al. Atypical memory B cells are greatly expanded in individuals living in a malaria-endemic area. J Immunol 2009;183:2176-82.

15 Knox JJ, Buggert M, Kardava L, et al. T-bet+ B cells are induced by human viral infections and dominate the HIV gp140 response. JCI Insight 2017;2.

16 Liu Y, Zhou S, Qian J, et al. T-bet+CD11c+ B cells are critical for antichromatin immunoglobulin $\mathrm{G}$ production in the development of lupus. Arthritis Res Ther 2017;19.

17 Wang S, Wang J, Kumar V, et al. IL-21 drives expansion and plasma cell differentiation of autoreactive CD11 chi T-bet $^{+}$B cells in SLE. Nat Commun 2018:9.

18 Hao Y, O'Neill P, Naradikian MS, et al. A B-cell subset uniquely responsive to innate stimuli accumulates in aged mice. Blood 2011;118:1294-304.

19 Rubtsov AV, Rubtsova K, Fischer A, et al. Toll-like receptor 7 (TLR7)-driven accumulation of a novel $C D 11 \mathrm{C}^{+} \mathrm{B}$-cell population is important for the development of autoimmunity. Blood 2011;118:1305-15.

20 Rubtsova K, Rubtsov AV, van Dyk LF, et al. T-box transcription factor T-bet, a key player in a unique type of B-cell activation essential for effective viral clearance. Proc Natl Acad Sci U SA 2013;110:E3216-24.

21 Rubtsova K, Rubtsov AV, Thurman JM, et al. B cells expressing the transcription factor T-bet drive lupus-like autoimmunity. J Clin Invest 2017;127:1392-404.

22 Sanz I. Systemic lupus erythematosus: extent and patterns of off-label use of rituximab for SLE. Nat Rev Rheumatol 2016;12:700-2.

23 Naradikian MS, Myles A, Beiting DP, et al. Cutting edge: IL-4, IL-21, and IFN- $\gamma$ interact to govern T-bet and CD11c expression in TLR-Activated B cells. J Immunol 2016:197:1023-8.

24 Russell Knode LM, Naradikian MS, Myles A, et al. Age-Associated B Cells Express a Diverse Repertoire of $V_{H}$ and $V_{\kappa}$ Genes wit Somatic Hypermutation. J Immunol 2017:198:1921-7

25 Colonna-Romano G, Bulati M, Aquino A, et al. A double-negative (IgD-CD27-) B cell population is increased in the peripheral blood of elderly people. Mech Ageing Dev 2009;130:681-90

26 Subramanian A, Tamayo P, Mootha VK, et al. Gene set enrichment analysis: a knowledge-based approach for interpreting genome-wide expression profiles. Proc Natl Acad Sci U S A 2005:102:15545-50.

27 Portugal S, Tipton CM, Sohn H, et al. Malaria-associated atypical memory B cells exhibit markedly reduced B cell receptor signaling and effector function. Elife 2015;4. doi:10.7554/eLife.07218. [Epub ahead of print: 08 May 2015]

$28 \mathrm{Chi} \mathrm{H}$. Regulation and function of mTOR signalling in T cell fate decisions. Nat Rev Immunol 2012;12:325-38.

29 Copp J, Manning G, Hunter T. TORC-specific phosphorylation of mammalian target of rapamycin (mTOR): phospho-Ser2481 is a marker for intact mTOR signaling complex 2. Cancer Res 2009:69:1821-7.

$30 \mathrm{Xu} \mathrm{W}$, Zhang JJ. STAT1-dependent synergistic activation of T-bet for IgG2a production during early stage of B cell activation. J Immunol 2005;175:7419-24.

31 Powell JD, Pollizzi KN, Heikamp EB, et al. Regulation of immune responses by mTOR. Annu Rev Immunol 2012;30:39-68.

32 Heidt $\mathrm{S}$, Roelen DL, Eijsink C, et al. Effects of immunosuppressive drugs on purified human B cells: evidence supporting the use of MMF and rapamycin. Transplantation 2008:86:1292-300.

33 Jenks SA, Cashman KS, Zumaquero E, et al. Distinct effector B cells induced by unregulated Toll-like receptor 7 contribute to pathogenic responses in systemic lupus erythematosus. Immunity 2018:49:725-39.

34 Burton AR, Pallett LJ, McCoy LE, et al. Circulating and intrahepatic antiviral B cells are defective in hepatitis B. J Clin Invest 2018;128:4588-603. 


\section{Systemic lupus erythematosus}

35 Nutt SL, Hodgkin PD, Tarlinton DM, et al. The generation of antibody-secreting plasma cells. Nat Rev Immunol 2015;15:160-71.

36 Jones DD, Gaudette BT, Wilmore JR, et al. mTOR has distinct functions in generating versus sustaining humoral immunity. J Clin Invest 2016;126:4250-61.

37 Wherry EJ, Kurachi M. Molecular and cellular insights into T cell exhaustion. Nat Rev Immunol 2015;15:486-99.

38 Wang C, Singer M, Anderson AC. Molecular Dissection of CD8 ${ }^{+} \mathrm{T}$-Cell Dysfunction. Trends Immunol 2017:38:567-76.

39 Doedens AL, Phan AT, Stradner MH, et al. Hypoxia-inducible factors enhance the effector responses of CD8(+) T cells to persistent antigen. Nat Immunol 2013;14:1173-82.

40 Wherry EJ, Ha S-J, Kaech SM, et al. Molecular signature of CD8+ T cell exhaustion during chronic viral infection. Immunity 2007;27:670-84.

41 Düvel K, Yecies JL, Menon S, et al. Activation of a metabolic gene regulatory network downstream of mTOR complex 1. Mol Cell 2010;39:171-83.

42 Wang R, Dillon CP, Shi LZ, et al. The transcription factor Myc controls metabolic reprogramming upon T lymphocyte activation. Immunity 2011;35:871-82.
43 Cho SH, Raybuck AL, Stengel K, et al. Germinal centre hypoxia and regulation of antibody qualities by a hypoxia response system. Nature 2016;537:234-8.

44 Fernandez D, Bonilla E, Mirza N, et al. Rapamycin reduces disease activity and normalizes $T$ cell activation-induced calcium fluxing in patients with systemic lupus erythematosus. Arthritis Rheum 2006;54:2983-8.

45 Lai Z-W, Kelly R, Winans T, et al. Sirolimus in patients with clinically active systemic lupus erythematosus resistant to, or intolerant of, conventional medications: a singlearm, open-label, phase 1/2 trial. Lancet 2018;391:1186-96.

46 Tipton CM, Fucile CF, Darce J, et al. Diversity, cellular origin and autoreactivity of antibody-secreting cell population expansions in acute systemic lupus erythematosus. Nat Immunol 2015;16:755-65.

47 Victora GD, Nussenzweig MC. Germinal centers. Annu Rev Immunol 2012;30:429-57.

48 Yurasov S, Wardemann H, Hammersen J, et al. Defective B cell tolerance checkpoints in systemic lupus erythematosus. J Exp Med 2005;201:703-11. 